Voix et Images

\title{
Délégués du Panthéon au plateau Mont-Royal : Sur deux romans de Michel Tremblay
}

\section{Antoine Sirois}

Volume 7, numéro 2, hiver 1982

Michel Tremblay

URI : https://id.erudit.org/iderudit/200322ar

DOI : https://doi.org/10.7202/200322ar

Aller au sommaire du numéro

Éditeur(s)

Les Presses de l'Université du Québec

\section{ISSN}

0318-9201 (imprimé)

1705-933X (numérique)

Découvrir la revue

\section{Citer cet article}

Sirois, A. (1982). Délégués du Panthéon au plateau Mont-Royal : Sur deux romans de Michel Tremblay. Voix et Images, 7(2), 319-326.

https://doi.org/10.7202/200322ar d'utilisation que vous pouvez consulter en ligne.

https://apropos.erudit.org/fr/usagers/politique-dutilisation/ 


\title{
Délégués du Panthéon au plateau Mont-Royal : Sur deux romans de Michel Tremblay
}

\author{
par Antoine Sirois
}

La critique a rapidement relevé, dans les deux premiers romans ${ }^{1}$ de la série des Chroniques du Plateau Mont-Royal de Michel Tremblay, l'apparition de personnages qu'elle a qualifiés d'imaginaires, de fantastiques ou de mythiques. Grand admirateur de la tragedie grecque ${ }^{2}$, l'auteur se devait peut-être un jour d'introduire dans le monde réaliste auquel il nous avait habitués des éléments mythiques. Pouvons-nous les identifier, relever des références dans le texte? Comment celles-ci s'inscrivent-elles dans les composantes du récit? Nous étudierons en premier lieu La Grosse femme d'à côté est enceinte.

Relevons d'abord un aspect symbolique qui nous conduit au plan mythique. La fonction du printemps paraît assez évidente à la première lecture du récit qui se déroule dans une seule journée, le 2 mai 1942, la «première grande journée du printemps ${ }^{3}$ dans la ville. Cette saison de renouvellement de la nature évoque les multiples naissances qui se préparent sur la rue Fabre et la re-naissance de plusieurs personnages. Elle provoque même la transformation que vivent plusieurs des futures mères et de nombreux voisins qui se sentent frustrés, "pognés": après la morte saison, un début de libération, de défoulement, d'épanouissement, d'espoir, de sympathie pour les autres, de communication. Du petit Marcel à la grandmère Victoire, les protagonistes connaissent pour la plupart, en une journée de soleil et de chaleur, une amélioration de leur situation. Richard, engoncé dans des conflits affectifs, trouvera une explication libérante à ses problèmes de puberté, Albertine, frustrée et jalouse du bonheur de sa belle-sœur, établira un lien de sympathie avec elle, la grosse femme, méprisée de son entourage dans sa grossesse tardive, trouvera la compréhension, et ainsi de suite.

La dimension mythique du récit n'est pas livrée au premier abord. La présence du chat Duplessis et du chien Godbout qui pensent et qui parlent étonne, mais les humains semblent tous vaquer normalement à leurs occupations quotidiennes. Les quatre “tricoteuses", Florence, Rose, Violette et Mauve, comme de bonnes québécoises, fabriquent, assises sur 
leur balcon, des pattes pour les bébés qui naîtront bientôt chez les voisines, tout en rappelant d'autres belles journées de printemps qu'elles situent selon les années de naissance. Elles apparaissent dès la première ligne de l'histoire et elles occuperont d'ailleurs le dernier paragraphe. Le lecteur constate que, travaillant, les yeux baissés sur leur laine bleue, elles obéissent à un certain devoir: " on est ben obligées, ça fait partie de nos arrangements!» 4 Devoir est même nécessité, apprend-on plus loin : “Faut jamais défaire ce qui est fait... Faut jamais retourner en arrière. On est là pour que tout aille vers l'avant. Ce qui est tricoté est tricoté, même si c'est mal tricoté ${ }^{5}$. Ce rôle inéluctable revêt une sorte de pérennité. Elles se souviennent des années de naissance, ces trois célibataires et leur mère, de leur vieille voisine et même de la mère de cette vieille voisine ${ }^{6}$. Mauve s'exclame enfin, devant cette mémoire qui semble inhabituelle : "Oui, on a toujours été là, Rose. Pis on s'ra toujours là. Tricote. Arrête pas. On est là pour ça” 7 .

Au déroulement du texte, après plus de cent pages, nous prenons plus clairement conscience que les quatre infatigables «tricoteuses", qui parlent «joual " comme leurs voisins, vivent en marge du commun, même si elles, paraissent bien intégrées. Elles peuvent remonter dans l'histoire des différentes générations des familles de la rue, mais elles sont aussi parallèles à leur temporalité : “parallèles à tout cela, enjambant les générations, catinant et tricotant tout ce temps, gardiennes cachées, surveillant, veillant, liées, protégeant de loin les berceaux, comptant les naissances mais non les morts, heureuses? Heureuses. Elles qui tricotent patiemment le temps $"{ }^{8}$. Parallèles, connaissant le passé, sachant "tout ce qui allait venir", ${ }^{9}$ elles sont de plus invisibles, sauf pour un jeune enfant innocent, Marcel, et pour quelques privilégiés. Comme l'avait déjà dit Florence : «Les chats peuvent nous voir. Les chats, pis des fois les fous ${ }^{10} \%$. L'aïeule Victoire, qui craint pour sa lucidité, les voit apparaître et disparaître.

On aura reconnu les trois Parques (latines) ${ }^{11}$ ou les Moïra (grecques) et leur mère, la Nuit, les divinités du Destin. Bien qu'au gré de l'évolution de la mythologie quelques variantes se soient introduites dans le rôle des Parques, elles sont perçues comme fileuses, tissant la trame de la vie, réglant sa durée depuis la naissance jusqu'à la mort. L'une file, l'autre enroule, l'autre coupe. Tremblay les a admirablement transformées, les yeux baissés sur leur laine, en "tricoteuses", incorporées dans la vie quotidienne de la rue Fabre, veillant aux naissances surtout. Habitant elles aussi la campagne à l'époque agraire, elles avaient déménagé comme leurs voisins en ville. L'auteur retient surtout la tradition ancienne touchant les Paŕques: "Ce furent probablement à l'origine des divinités de la naissance, mais la spéculation ultérieure les a élevées jusqu'à la personnification du destin » 12

Si elles tricotent d'abord pour la vie, elles peuvent aussi intervenir dans la mort. Violette, par erreur, a fermé sa dernière patte : «J'ai tricoté la boule parfaite ${ }^{13}$. Un protagoniste verra son cycle accompli. On peut craindre pour le chat Duplessis qui a joué avec la balle de laine que Vio- 
lette avait échappée et s'était vu infliger des blessures mortelles par Godbout. Florence, la mère, pleine d'appréhension, avait mis la patte refermée dans sa poche et demandé à sa fille de réparer son erreur. Celle-ci avait ramassé le chat malade. Ce qui devait arriver, arriva, mais ce fut Ti-Lou, la Louve d'Ottawa, qui mourut et en poussant un cri tel que Florence sortit la boule de laine de sa poche et regarda Violette en disant: "C'tait pas pour Duplessis... t'a tricoté la mort de quelqu'un d'autre» ${ }^{14}$. Violette Athropos avait tranché le fil de la destinée, sans retour.

C'est à Florence que le petit Marcel avait confié le chat mourant, son ami. On le retrouve, a la toute fin de l'histoire, sur ses genoux. Elle le protège du chien qui l'avait poursuivi, et réussit à l'endormir, dans la noirceur. La Nuit alias Florence ne semble en fait bien fonctionner que dans le noir. Elle ne contera l'histoire des générations qu'à ce moment. « II fallait attendre au soir, à l'heure du coucher, dans le noir le plus complet, avec pour seul témoin l'œil crevé de la lune, pour parler... Là seulement. elle pourrait s'asseoir à côté du lit de sa fille et raconter »15. Elle a naturellement la lune pour compagne. Quand Josaphat invitera Marcel à respecter la lune toute sa vie, parce qu'elle est "la seule chose au monde dont tu peux être sûr » ${ }^{16}$, Florence, qui a écouté à sa fenêtre, reçoit un salut du conteur. Elle y répond avec un "sourire complice" 17. La tradition mythologique voit dans la nuit et dans la lune des symboles de fertilité, de fécondité. La nuit, principe féminin, précède la création de tout. La lune, maîtresse de toutes les choses vivantes, a tissé les destins et c'est ainsi que les Parques sont considérées comme divinités lunaires ${ }^{18}$. Nous comprenons mieux maintenant, dans ce roman consacré à la maternité (librement consentie) et à une certaine régénération des protagonistes en ce jour de printemps, la présence des Parques, de la Nuit et de la Lune qui viennent soutenir, achever et universaliser le thème central. Nous saisirons peut-être mieux, incidemment, l'intégration au roman d'un conte folklorique de dix pages sur l'allumeur de la lune.

Les forces de vie agissent dans cette histoire. Les forces maléfiques y travaillent également. Elles ont faces d'animal et d'homme. Si nous relevons en Duplessis, le chat et en Godbout, le chien, personnalisés par l'auteur, une référence à la vie politique des années quarante, nous pouvons aussi détecter dans le comportement de ce molosse poussif et laid, une incidence mythique. On nous le présente d'abord comme un animal «intraitable et même vicieux" 19 , aux "dents absolument terrifiantes" 20 . II entretient des idées de carnage et d'assassinat contre Duplessis. Après la bataille “mortelle " qu'il lui a livrée, il veut s'assurer qu'il est bien mort ${ }^{21}$. Même si ceci ne sort pas trop d'une relation courante entre chien et chat, on perçoit quand même qu'il s'agit là d'une bête peu commune, terrifiante et maléfique, surtout si elle est reliée aux espaces infernaux, comme c'est le cas. Elle jappe «comme la totalité des démons de l'enfer " 22 et elle est bien déterminée à pousser Duplessis dans “l'enfer des chats" ${ }^{23}$. Ne pensonsnous pas ici à Cerbère, le chien monstrueux qui monte la garde aux portes 
de Hadès, du royaume de la Mort? Nous pouvons peut-être encore ne voir en ces textes que des images ou des comparaisons. L'association entre le rôle de Godbout et celui de Gérard Bleau viendra confirmer notre déduction. En cette " coqueluche de la rue Dorion, le don Juan de la côte Sherbrooke" ${ }^{24}$, Thérèse (11 ans) découvre l'homme auquel elle aspirait dans sa sensualité précoce. Elle tourne autour de lui et s'en approche comme une “araignée-chatte aux désirs confus» 25 , et, finalement, l'embrasse. Celui-ci est totalement chaviré par ce "geste irrévocable qui coupe sa vie en deux parties distinctes: celle d'avant la petite fille, celle d'après" 26 . «Elle lui avait fait entrevoir des horizons barioles du rouge de la honte et du noir du péché, ce qui l'excitait énormément et donnait au danger un air de fête macabre, de cérémonie invitante et secrète où régnaient en maîtres absolus la culpabilité, la dissimulation, le remords ${ }^{27}$. Quand elle quitte le parc, il la poursuit, comme Godbout traque en même temps Duplessis, jusque sur la rue Fabre ${ }^{28}$ et, terré dans l'ombre, il la guette comme Godbout le fait pour le chat. Ils sont étroitement associés dans leur guet: «Godbout et Gérard Bleau n'avaient pas bougé" ${ }^{29}$. Ils sont liés dans un même projet meurtrier. Thérèse, sur son balcon, apeurée, se demande ce que Bleau veut faire et se voit dire par son cousin Richard “Mais, j'sais c' qu'y veut, moé... $y^{\prime}$ attend sa victime pour la tuer... " ${ }^{30}$. Ces deux êtres semblent bien incarner les forces du mal et de la mort. Si le chien a été associé à l'enfer, Bleau l'est aussi, car il est qualifié «d'ange déchu» ${ }^{31}$ par le narrateur.

Ces allusions à la mythologie, cette évocation de forces supérieures qui président au destin des humains et qui sont des explications imagées que se donnent les générations pour expliquer les phénomènes qui les dépassent, ajoutent à la richesse d'une œuvre. L'histoire présente, trouvant ses assises dans la vie humble de la rue Fabre, rejoint les problèmes fondamentaux de l'homme : la vie, la mort, le bien, le mal, l'amour.

Le second roman marque à la fois la continuité et la discontinuité. Les tricoteuses sont toujours à "leur éternel tricot» ${ }^{32}$, mais le narrateur n'y fait qu'allusion. On constate un déplacement majeur: toujours invisibles, sauf pour quelques privilégiés, elles dévoilent une facette nouvelle de leur rôle. Florence, qui avait raconté quelques générations de la famille de la grand-mère Victoire, remonte maintenant dans son récit jusqu'aux ancêtres français et aux premiers temps de la colonie, et, avec ses trois filles, plonge le petit-fils Marcel “dans les légendes de la chasse-galerie" 33, «dans les histoires de la bête Pharamine " ${ }^{34}$, comme elle le faisait autrefois, au village de Duhamel, pour l'oncle Josaphat. Les trois sœurs reprennent aussi pour le petit citadin d'aujourd'hui leur mission d'initiatrices aux arts, Mauve en musique, Violette en chant et Rose en poésie, comme le précise le narrateur ${ }^{35}$. Elles qui n'avaient pas trouvé de disciples dans la première génération urbaine, se montrent tout heureuses de poursuivre leur tâche : "Mais ça fait plaisir de voir que quelqu'un de la nouvelle génération a besoin de nous autres..." ${ }^{36}$. Marcel, que sa famille considère comme un poète ou un fou (et que Tremblay voit avec son chat au cœur même des Chroniques) ${ }^{37}$, 
est initié aux grandes consolations de la musique par Mauve au piano. Josaphat, jeune, l'était par le violon. Comme Marcel n'a que quatre ans, Florence lui dit : "Plus tard, quand tu seras plus grand, quand tu pourras en comprendre plus, j'te dirai les grandes compensations que peuvent te prodiguer Violette et Rose, mais là t'es trop petit. La musique devrait te suffire pour le moment ${ }^{38}$. Les allusions du narrateur sont ici plus transparentes et les références, faciles. Nous songeons aux Muses. Déesses de la mémoire capables de prophétie, elles partagent ces dons avec les Parques. Mais c'est surtout comme inspiratrices poétiques qu'elles se manifestent dans le présent roman. La légende leur a même assigné à chacune une fonction déterminée que l'on retrouve en partie chez nos quatre femmes: inspiration de l'histoire par Clio (Florence), inspiration de la musique par Euterpe (Mauve), inspiration de la poésie lyrique et amoureuse par Terpsichore et Erato (Rose). Si les Parques présidaient dans le premier roman au destin de l'ensemble, les Muses ne s'adressent qu'aux «happy few " pour les initier à la beauté : "la chance qu’ils avaient eux, Victoire, Marcel et lui-même, Josaphat-le-Violon de pouvoir goûter à tout ça alors que les autres étaient condamnés à la médiocrité de leur vie quotidienne " ${ }^{39}$. Dans le premier récit, les références aux divinités adjuvantes s'inscrivaient dans la thématique centrale de la maternité, dans le second, elles s'intègrent au thème de la tradition historique et folklorique.

A la fin du premier roman, nous trouvions le chat Duplessis sur les genoux de Florence. Elle l'avait soustrait au chien infernal Godbout qui l'avait massacré et le guettait toujours, mais ne voyait qu'un balcon vide. Invisibles étaient les femmes, invisible était aussi devenu le chat, comme le second roman nous permet de le constater. Seuls ceux qui voient les femmes voient le chat qui garde, malgré tout, un comportement félin sur la rue Fabre et s'exprime comme ses habitants. Mais était-il né d'hier? Marcel découvrait la beauté de la musique à travers la sonate de Mauve, Duplessis, lui, «semblait (la) retrouver dans le fond de sa mémoire "40. II semble rejoindre les femmes dans leur pérennité et il leur est associé dans leur mission future auprès de Marcel. II «va continuer ton éducation partout où tu vas aller " ${ }^{41}$, lui dit Florence. II lui enseignera le vocabulaire. Faut-il voir dans la présence et les agissements de ce personnage associé aux divinités une référence à la mythologie? Le rapprochement que nous pouvons suggérer ne peut être puisé dans l'Olympe qui ne semble pas contenir de dieu chat. Nous trouvons dans le panthéon égyptien une divinité plausible, la déesse Bastet, évoquée comme bienfaitrice et protectrice de l'homme, susceptible de se joindre aux Muses dans l'éducation de Marcel parce qu'elle était déesse du plaisir et aimait la musique et la danse. Elle peut aussi faire bon ménage avec les divinités lunaires que sont les Parques parce que les Égyptiens associaient le chat à la lune. Une difficulté s'ajoute dans la signification. L'apparition avec Marcel du chat devenu invisible soulève chez Victoire une réaction profonde: «Va-t'en, maudit chat!... Maudit fantôme! Maudite mort!»42. Elle ajoute pour Marcel : «Tu transportes ma mort dans tes bras » $^{43}$ À la fin du récit, elle aperçoit encore 
«le maudit chat porteur de mort» ${ }^{44}$. Duplessis n'est pas noir, mais tigré. Victoire réagirait plus à l'apparence fantomatique du chat disparu du monde des êtres visibles comme elle avait été troublée, dans l'autre roman, par l'apparition de Florence dans une maison qu'elle croyait inhabitée, craignant pour sa lucidité. Si l'on ramène Duplessis au niveau des humains, on pourrait aussi voir en lui Mentor, l'ami d'Ulysse qui lui confie l'éducation de Télémaque, et en qui se déguisait Athena pour les secourir tous les deux. Comme on le constate, dans ce cas-ci, un manque d'allusions suffisamment précisées nous oblige à la spéculation.

C'est plutôt le chien Godbout, que nous avons associé à Cerbère empêchant dans l'Hadès les âmes mortes de retourner à un monde supérieur vers un salut encore possible, qui évoque, avec Gérard Bleau, le mal et la mort. Il a perdu dans ce roman toute importance, mais dans les deux allusions qui lui sont faites, nous pouvons trouver confirmation aux précédentes interprétations. Albertine sondant la maison inhabitée où se rend Marcel et où le chat a été déposé, se voit regardée curieusement par "un vieux chien, vautré dans un carré de terre où poussait quelques plants de salade du diable "45. Duplessis, plus tard, ira vérifier si Godbout “régnait toujours dans la terreur et dans le sang "46. Gérard Bleau, qui traque toujours, dans l'inquiétude et la torture, le remords et la culpabilité, la fillette de onze ans, Thérèse, pour la violer, accroît sa présence dans le second roman, mais perd son association au chien infernal qui rehaussait le caractère abject de sa quête. Les forces du mal et de la mort, humaines et supra-humaines, étaient en quelque sorte liguées dans le premier récit, ce qui conférait une dimension mythique au conflit avec les forces de vie. Le seul renvoi au caractère diabolique du rôle du Don Juan se trouve dans une allusion du narrateur qui précise que Bleau avait pour les femmes de son quartier «un petit goût d'enfer mal fermé »47. La contrepartie ou le pendant inversé de la mère glorifiée du premier récit, la marâtre, mère Benoîte-desAnges, constitue la force agissante majeure et néfaste du second. Elle n'écope que d'un surnom, "mère Dragon du Yable», sans autre rappel d'un univers maléfique. Le narrateur, cependant, à l'occasion de la poursuite de Gérard Bleau, introduit un être transcendant, relevant de la tradition vétérotestamentaire transmise dans l'enseignement chrétien de l'époque, le "Dieu Vengeur» ${ }^{48}$. II vient, sous la forme du soleil, harceler le coupable: "le soleil le frappait au front comme s'il s'était soudain trouvé dans le champ de vision de Dieu et la gravité de ce qu'il se préparait à faire à cette enfant trop belle... lui apparaissait dans toute son horreur "49. Même si cette intervention divine, qui n'est pas sans rappeler l'œil qui poursuivait Caïn, dans le poème de Victor Hugo, va à l'encontre d'un geste que le narrateur lui-même réprouve, le Dieu “qui était descendu de ses lointains domaines " 50 n'est pas considéré comme bénéfique ${ }^{51}$ et n'est pas associé aux autres divinités protectrices.

Avant la parution du troisième et même du quatrième roman prévus dans les Chroniques du Plateau Mont-Royal, il n'est pas possible d'en arriver à des conclusions exhaustives et plus définitives. Nous avons constaté 
une intégration plausible et heureuse de personnages mythologiques dans le quotidien de la rue Fabre. Nous avons réussi à identifier un certain nombre de références et d'allusions à la mythologie. D'autres clarifications viendront sans doute s'ajouter. Dans la typologie retenue par la mytho-critique ${ }^{52}$, nous pouvons déja affirmer qu'il ne s'agit pas de romans mythiques, qui racontent à nouveau un mythe connu, mais peut-être de romans mythologiques, avec références à des récits mythiques ou à des personnages mythologiques, mais encore faudra-t-il nuancer et préciser. Restera aussi à apprécier si certains récits mythiques sur la quête du héros, les rites de passage, par exemple, ont fourni un pattern qui structure l'œuvre. Par rapport à la thématique, nous pouvons établir, sur le rôle des personnages, quelques comparaisons entre les deux romans. Dans le premier, les divinités adjuvantes sont très présentes et actives, supportant la thématique centrale de la fécondité et de l'amour conjugal, participant au destin de la collectivité. Dans le second, elles interviennent pour un thème important, touchant aussi à la tradition québécoise, mais secondaire par rapport au thème de la contestation de l'ordre clérical en place et des amitiés sous toutes leurs formes, et elles ne le font que pour quelques privilégiés. Du déploiement des forces bénéfiques et maléfiques du premier récit, nous tirions l'impression d'un combat "épique" pour un enjeu touchant les valeurs fondamentales du bien et du mal, de la vie et de la mort. Les interventions plus ponctuelles et diversifiées des personnages transcendants, ainsi que moins conjuguées et importantes pour l'ensemble, réduisent leur impact dans le second récit et par conséquent sa dimension mythique.

Attendons la suite, mais il nous a paru que la mytho-critique pouvait déjà apporter une contribution utile à la compréhension et à l'appréciation de ces Chroniques importantes et étonnantes de Michel Tremblay.

1. Michel Tremblay, La Grosse Femme..., Montréal, Léméac, 1978. Thérèse et Pierrette..., Montréal, Leméac, 1980.

2. Interview avec Martial Dassylva : "Une pièce inspirée de la tragédie grecque", la Presse, Montréal, 13 mai 1978, p. D-1.

3. Michel Tremblay, op. cit., p. 61.

4. Op. cit., p. 53.

5. Op. cit., p. 101

6. Op. cit., p. 102.

7. Op. cit., p. 103.

8. Op. cit., p. 206-7.

9. Op. cit., p. 207.

10. Op. cit., p. 108.

11. Quatre critiques ont identifié les Parques: Jacques Ferron, «Le nouveau né de M. Tremblay", La Nouvelle du Haut Saint-François, 30 janvier 1979, p. 20; Robert Mélançon, “Michel Tremblay romancier ", le Devoir, 4 novembre 1978; André Vanasse "Le chroniqueur de la ville et le chroniqueur des champs", Lettres québécoises, no 13, février 1979, p. 11; et Jacques Allard, “La Grosse Femme..., voix et images, vol. IV no 3, p. 537.

12. Mircéa Eliade, Traité d'histoire des religions, Paris, Payot, 1959, p. 163-164.

13. Michel Tremblay, op. cit., p. 208. 
14. Op. cit., p. 283.

15. Op. cit., p. 205.

16. Ibid., p. 293-94.

17. Ibid., p. 294.

18. Voir en plus du traité ci-haut mentionné de Mircéa Eliade, les dictionnaires consacrés à la mythologie et aux symboles comme ceux de Pierre Lavedan, Robert Graves, etc.

19. Ibid., p. 156.

20. Ibid., p. 157.

21. Ibid., p. 322.

22. Ibid., p. 157.

23. Ibid., p. 322.

24. Wbid., p. 244 :

25. Ibid., p. 185.

26. Ibid., p. 188.

27. Ibid., p. 247.

28. Ibid., p. 322.

29. Ibid., p. 323.

30. Ibid., p. 323.

31. Ibid., p. 252.

32. Ibid., p. 349 .

33. Ibid., p. 206.

34. Ibid., p. 206.

35. Ibid., p. 349.

36. Ibid., p. 221.

37. Réginald Martel, "Michel Tremblay : Thérèse et Pierrette à l'école des SaintsAnges : une belle trinité de filles dans l'ombre d'un Dieu muet», la Presse, Montréal, 25 octobre 1980, p. C-1.

38. Michel Tremblay, op. cit., p. 220.

39. Op. cit., p. 350.

40. Michel Tremblay, Thérèse et Pierrette à l'école des Saints-Anges, Montréal, Leméac, 1980, p. 219.

41. Ibid., p. 220.

42. Ibid., p. 275.

43. Ibid., p. 275.

44. Ibid., p. 363.

45. Ibid., p. 193.

46. Ibid., p. 299.

47. Ibid., p. 179.

48. Ibid., p. 179.

49. Ibid., p. 203.

50. Ibid., p. 203.

51. Voir aussi : Réginald Martel, art. cité no 37.

52. John White, Mythology in the Modern Novel, Princeton University Press, 1971 ; "Myths and Patterns in the Modern Novel", Mosaic, 43, Spring 1969.

E.W. Nerd, "Myth Criticism, Limitations and Possibilities", Mosaic, 43, Spring 1969. 